\title{
SIBILLA ALERAMO. UNA NUOVA DONNA
}

\section{SIBILLA ALERAMO. A NEW WOMAN}

\author{
Valentina Zucchi \\ Universidad Complutense de Madrid, Madrid, España \\ vzucchi@ucm.es
}

Recibido: octubre de 2021

Aceptado: noviembre de 2021

Parole chiave: Sibilla Aleramo, femminismo, Una donna, maternità, nuova identità.

Key words: Sibilla Aleramo, A Woman, feminism, maternity, new identity.

Riassunto: Nella cornice di una realtà storico-sociale fortemente ostile per le donne italiane del primo Novecento, molte voci femminili si ribellano scrivendo testi di denuncia sociale in cui il nemico è sempre il sistema patriarcale-misogino che riversa la sua violenza sulle donne. Tra queste voci urla con grande potenza quella di Sibilla Aleramo, la cui lotta femminista narrata nel suo romanzo autobiografico Una donna, dà impulso a un percorso esistenziale verso la conquista di una nuova identità. Nasce una nuova donna che ha saputo ascoltare la propria legge rinunciando perfino al figlio: il sacrificio umano per la libertà.

Abstract: In the framework of a socio-historical reality which was strongly hostile to Italian women in the early twentieth century, many rebellious female voices express their dissent in works of social denunciation in which the enemy is always the patriarchal-misogynist system that discharged its violence onto women. One of these voices cries out powerfully, that of Sibilla Aleramo, whose feminist struggle was narrated in her autobiographical romance, A Woman, giving rise to an existential path towards the achievement of a new identity. A new woman was born, capable of heeding her own law, even relinquishing her own son: a human sacrifice for freedom.

\section{Contesto storico e sociale dell'Italia del primo Novecento: situazione delle donne e processo di emancipazione femminile}

Sviluppatosi intorno alla svolta del secolo, il movimento delle donne italiane rimase piccolo e diviso, nella misura in cui i suoi membri, più che scendere in piazza e impegnarsi in un movimento militante, si dedicavano alle opere sociali in favore di donne e bambini indigenti.

Sibilla Aleramo e alcuni altri personaggi di primo piano, quali Teresa Labriola o Ersilia Majno, tendevano in generale a distinguere la storia del femminismo in due fasi, o per meglio dire, in due movimenti: I'uno di carattere estremamente individualistico, spes- 
so arditamente rivoluzionario, l'altro teso, insieme alla conquista dei diritti, alla rigenerazione morale della società attraverso l'affermazione dello "spirito femminile autonomo" e della nuova "etica femminile", scaturiti da una nuova visione del materno come potenza e come segno della superiorità morale della donna (Buttafuoco, 1988: 11). Questa visione del femminismo moderno corrisponde pienamente al sentire di Sibilla Aleramo, la quale riunisce in sé i due movimenti dell'emancipazionismo: da un lato la missione morale della rigenerazione della società di cui si avvale la sua scrittura, ovvero, il tentativo di cambiare la comunità delle donne e di insegnare loro qualcosa di buono attraverso le sue parole, dall'altro un'idea di femminismo lontano sia dalla militanza che da forti teorie ideologiche ma che diventa invece una spinta individuale, una rivoluzione personale in cui viene messo in gioco anche il senso della maternità che viene ripensata, ridefinita $\mathrm{e}$ liberata da vecchi condizionamenti e che diventa la risposta a un femminismo assolutamente moderno.

II movimento femminista italiano era debole e ciò che contribuiva a renderne difficile una compattezza era anche la presenza della chiesa cattolica, la quale si mantenne irriducibilmente ostile al sistema liberale; la sua cultura antimodernistica, che in genere mal tollerava le filosofie individualistiche, era contraria all'emancipazione femminile. Le donne si trovavano inoltre in una situazione di esclusione, non solo dalla maggior parte degli atti giuridici e commerciali in assenza del consenso dei propri mariti, ma anche dalla possibilità di agire come tutrici nei confronti dei figli ${ }^{1}$ (Duby e Perrot, 2007: 147).

1. Sibilla Aleramo infatti non riuscirà mai ad ottenere l'affidamento del figlio dal quale si separò quando aveva sette anni e che rimarrà per sempre
Inoltre, anche il lavoro delle donne, lontano dall'essere premiato, avveniva in sordina, in una situazione di svantaggio sociale rispetto a quello esercitato dagli uomini. Va ricordato però, che a quei tempi, il movimento femminista continuava a rimanere poco unito e raramente combattivo ed era fiancheggiato dalle élite liberali che favorivano gli atteggiamenti antifemministi come per esempio negare alle donne il diritto al voto.

Nella società italiana dei primi del Novecento esisteva una separatezza molto forte tra mondo maschile e mondo femminile; questa situazione durò poi anche durante il patriarcato fascista; infatti quest'ultimo teneva per fermo che uomini e donne fossero per natura diversi. Esso politicizzò pertanto tale differenza a vantaggio dei maschi e la esasperò in un sistema particolarmente repressivo, completo e nuovo, inteso a definire i diritti delle donne come cittadine e a controllarne la sessualità, il lavoro salariato e la partecipazione sociale. Lo Stato si proclamava come l'unico arbitro della salute pubblica e, in linea di principio, esse non avevano nessun potere di decisione riguardo alla procreazione dei figli.

Il fascismo tentò di imporre le gravidanze proibendo l'aborto e la vendita di contraccettivi e contemporaneamente favorì gli uomini da tutti i punti di vista: del lavoro, all'interno della struttura familiare, del sistema politico e della società in generale. Le opinioni fasciste sulle donne erano impregnate di misoginia: "Le donne sono angeli o demoni, nate per badare alla casa, mettere al mondo dei figli e portare le corna" (Duby e Perrot,

con il padre. La legge non la protesse e la scrittrice rivide il figlio solo una volta quando questi era già grande. Egli non volle mai più saperne della madre. 
2007: 146-152). Lo stesso Mussolini e i suoi sostenitori dicevano di non discutere se la donna fosse superiore o inferiore all'uomo ma semplicemente diversa. La politica riproduttiva fu un tratto essenziale della politica sessuale del regime fascista con il suo relativo attacco alla libertà di riproduzione e il motto era sempre "nascite, ancora nascite"2 (De Giorgio, 1992: 111). In generale la maternità fascista fu ad alta intensità del lavoro: le donne partorivano numerosi figli e lavoravano molte ore al giorno e le loro condizioni di vita si basavano su enormi fatiche e scarsissimi riconoscimenti sociali e politici; le parole "sacrifici e stenti" riempirono i racconti femminili riguardanti la maternità e l'istinto materno.

\section{La violenza patriarcale}

Nella cornice della nostra storia di donne, che si è svolta nel seno di una società patriarcale e maschilista, i maltrattamenti e la violenza nei confronti delle donne sono sempre stati presenti nel corso della nostra esistenza; la violenza intesa come sopruso, umiliazione, controllo, manipolazione, mortificazione e sottomissione dell'altro sesso: la donna.

2. Virginia Woolf, la femmisista per eccellenza, nonché punto di riferimento per tutte le scrittrici coscienziate verso la costruzione di una nuova identità di donna, nel racconto Una società, inserito nella collana di racconti Un collegio di ragazze, dice: "Siamo andate avanti per secoli a supporre che gli uomini fossero ugualmente industriosi e che le loro opere avessero pari merito. Mentre noi partorivamo bambini, loro, abbiamo supposto, partorivano libri e i quadri. Noi abbiamo popolato il mondo. Loro lo hanno civilizzato. Ma adesso che sappiamo leggere, chi ci impedisce di giudicare i risultati? Prima di mettere al mondo un altro bambino dobbiamo giurare di scoprire com'è fatto, il mondo".
L'atteggiamento misogino prende la donna come il bersaglio da atteccare per mezzo di ingiurie e oltraggi e scatena l'odio irrazionale contro i rappresentanti più innocenti e indifesi dell'altro sesso (Martínez Garrido, 2009: 128). Le donne, spesso-vittime di angherie e di perverse macchinazioni psicologiche, nonché a volte anche di violenze fisiche da parte degli uomini frustrati e violenti, sopravvivono a simili drammi e resistono perché abituate ad osservare il repertorio di atteggiamenti morali quali la rassegnazione, la paura e l'accettazione di un castigo, che si tramanda di madre in figlia, di generazione in generazione (Folli, 2007: VIII).

Se la storia è piena di siffatte esperienze femminili, anche la letteratura, intesa come specchio di vita, diventa la testimonianza di una verità al femminile. Nella letteratura del primo Novecento sono tante le donne che decidono di scrivere per raccontare una storia di vita personale fatta di sofferenze e oppressioni.

Il tema della violenza di genere fino ad allora non veniva nemmeno considerato come un problema esistenziale femminile giacché le donne, nella maggior parte dei casi, tranne alcune che avevano già potuto conquistare una loro maturità, non erano ancora padrone di uno spazio di riflessione, di un tempo proprio; non esisteva il "tempo della solitudine" (Zambrano, 1988: 20) che potesse in qualche modo facilitare loro l'esercizio dell'introspezione e una saggia ed utile pratica dell'autocoscienza. II tempo della solitudine è quello che corrisponde all'uomo (inteso come essere umano) che si sente già individuo. Ma le donne non si sentivano "individui" perché non sapevano ancora muoversi attivamente nella storia; questo accadrà loro solo quando verrà accettata la responsabilità o quando la si 
riuscirà a vivere moralmente (Zambrano, 1988: 11).

Le donne, spesso inconsapevoli e quindi confuse, attonite, davanti ad un fatto di violenza subíto, si consegnavano inconsciamente al meccanismo della rimozione, intera o parziale, di quanto fosse successo loro; di conseguenza tutto ciò le rendeva incapaci di reagire, immobili nel buio della loro ignoranza.

Detto ciò, il tema della violenza fino a quel momento veniva dunque rappresentato più come un "topos letterario che non come una confessione di un vissuto esistenziale lacerante" (Martínez Garrido, 2009: 130), come accadrà invece nel momento in cui le donne si decidono a prendere in mano una penna per parlare della loro vita, dando inizio così a quella che sarà una letteratura che si allontana dal precedente romanzo rosa, ameno e adatto allo svago, per abbracciare una funzione ben precisa: quella di dire la verità, di creare un documento storico che sveli la realtà delle cose in una letteratura impegnata che coincide con la nascita di "una nuova sensibilità femminile che esprime un femminismo moderno, completamente femminile" (Folli, 2007: XIII).

L'elenco di donne scrittrici che decidono di raccontare il Vero e di svelare il dramma della loro vita è molto lungo, e in tutte commuove la forza morale di questi personaggi femminili che riescono, attraverso la scrittura che dà impulso a un viaggio intimo e solitario di autocoscienza e di liberazione, ad arrivare ad essere dei soggetti autonomi e nuovi dal punto di vista esistenziale e sociale.

Sibilla Aleramo, Neera, Annie Vivanti sono le protagoniste della prima metà del Novecento che scrivono per raccontare la loro storia; una tremenda storia di vita che contiene una forte e pungente denuncia nei confronti della società patriarcale e misogina del tempo e degli uomini maltrattatori che, tiranneggiando in piena libertà, umiliano la donna e la rilegano in un angolino del mondo, in un fitto silenzio.

La dolorosa e umiliante violenza psicologica e sessuale, a volte non detta ma implicitamente conosciuta, impregna le pagine di Duello d'anime di Neera, di Vae Victis di Annie Vivanti e di Una donna di Sibilla Aleramo.

Neera si separa dalle altre scrittrici, in quanto persegue un idealismo conservatore, seguendo un femminismo di tipo cattolico. La sua posizione è contraria ai tentativi di emancipazione femminile già presenti nella società italiana di quegli anni. Vale a dire, la scrittrice milanese si serve delle tematiche legate all'emarginazione femminile con lo scopo di mantenere ed elevare un prototipo ideale di donna, entro i confini del quale si innalzano come modelli, quasi in modo inverosimile, i comportamenti tipici della santità, dell'abnegazione, del sacrificio e del martirologio cristiano o paracristiano. Assolutamente diversa è la posizione di Sibilla Aleramo che in un certo modo può essere considerata il "pilastro del pensiero socialista, del pensiero utopistico e del femminismo di sinistra" (Martínez Garrido, 2009: 131).

I romanzi di queste tre scrittrici in ogni caso hanno come tema in comune quello della violenza del protagonista maschile nei confronti della donna. Questa violenza miticamente ancestrale, segue il mito della vergine perseguitata, sedotta ed abbandonata, presente in tante leggende, miti e fiabe popolari.

Nell'Ottocento, tante protagoniste del romanzo rosa e sentimentale, dopo aver so- 
fferto l'abuso sessuale, cercano di autoingannarsi e di camuffare la violenza patita giustificandola in nome di un amore-passione impulsivo dell'uomo che, "nell'ottica di una società misogina ed androcentrica" (Martínez Garrido, 2009: 135), le inganna con perverse arti maschili, con contorti stratagemmi che confondono le fragili ed innocenti vittime per poi privarle dell'unico valore che gli rimane: la verginità.

Invece nei romanzi di Aleramo, di Neera e di Annie Vivanti, il tema della violenza di genere mette l'accento sui tentativi emotivi e sulle conquiste psicologiche di autoaffermazione personale delle protagoniste che, da vittime passano ad essere, al termine del loro viaggio, soggetti eroici.

II tema della violenza contro le donne riempie le pagine della narrativa europea tra il XIX e il XX secolo, fino a diventare un tema ricorrente della letteratura femminile che testimonia una sofferta verità che fino a quel momento veniva accettata in silenzio. La letteratura, al di fuori dello spazio letterario egemone, si trasforma in un documento storico importantissimo della storia femminile.

Limitandoci al panorama letterario italiano delle scrittrici che inaugurano il filone di una letteratura di denuncia, a quelle già menzionate precedentemente, bisogna aggiungere anche Elsa Morante.

Sia Vae Victis di Annie Vivanti, del 1918, sia La Storia di Elsa Morante, scritto molto più avanti, nel 1974, trattano il tema della violenza sessuale patita dalle donne; le protagoniste dei loro rispettivi romanzi, subiscono uno stupro per mano dei soldati durante la prima guerra mondiale, nel caso del primo romanzo, e durante la seconda nel caso di La Storia.

In Vae Victis si tratta delle violenze sessuali sofferte dalle giovani belghe da parte dei soldati tedeschi. La protagonista rimarrà incinta dopo essere stata stuprata da un soldato e, dopo aver contemplato la possibilità di un aborto, deciderà di tenere il bimbo nonostante il mondo intero la umili, la emargini e non la sostenga in questa durissima scelta; la protagonista deciderà di portare avanti la gravidanza da sola e di amare il suo bimbo nonostante sia il frutto di una violenza devastante. Lo stesso succederà a Ida, la protagonista di La Storia di Elsa Morante. Questo personaggio femminile viene violentato da un soldato tedesco ubriaco in cerca d'amore nel 1941, in circostanze quasi improbabili per inverosimiglianza. Paralizzata dalla paura, la maestra, subisce la violenza del soldato e quasi vi si abbandona in un atteggiamento che fa proprio pensare alla protagonista di Una donna di Sibilla Aleramo. Anche quest'ultima infatti verrà a sua volta violentata da chi ne diventerà poi il marito, in una circostanza che la confonde a tal punto da metterla nelle condizioni di ricevere quel gesto senza opporvisi. Nell'opera di Morante, compiuto il misfatto, il soldato tedesco sparirà e Ida deciderà di portare avanti la gravidanza e di partorire e di crescere con immenso amore "il suo piccolo Useppe dagli occhi di mare violaceo e di cielo bavarese" (Morante, 1995: VIII) tra le rovine di una Roma devastata dalla guerra.

In queste storie di scempio umano e di violenza di genere le protagoniste sono comunque sospinte in avanti dalla forza della loro maternità che le fa sentire quasi invincibili.

La Storia venne colto come un romanzo di ribellione, di protesta; Cesare Garboli, nella sua prefazione al libro, lo definisce "II romanzo delle vittime, delle cavie che non sanno il perché della loro morte" (Morante, 1995: VIII). La stessa Morante definì il 
suo romanzo un manifesto, un'azione politica, così come lo fu Una donna che più avanti esamineremo.

Bisogna in tutti i modi sottolineare una grande differenza tra la scrittura di Sibilla Aleramo e quella delle altre scrittrici a lei contemporanee e appena menzionate; mentre queste ultime infatti creano invenzioni letterarie che sono al servizio del tipo di letteratura che perseguono, i drammi narrati da Aleramo sono invece autobiografici, vissuti personalmente sulla sua propria pelle, e ciò aggiunge naturalmente alle sue narrazioni una maggior carica di potere di coinvolgimento, che si deve non solo alla verità dei fatti raccontati, ma anche alla chiara urgenza della scrittrice di svelarne la dura realtà, nella necessità di creare un documento di denuncia sociale. Aleramo con il suo primo romanzo autobiografico Una donna (1906) ci offre un documento storico sulla problematica dell'essere donna nella società del primo Novecento che "testimonia una forte denuncia sociale sulla netta disuguaglianza tra uomini e donne" (González, 2009: 293).

\section{Sibilla Aleramo, Una donna: Manifesto del femminismo italiano}

Una donna, l'opera più importante di Sibilla Aleramo (Marta Felicina Faccio, Rina, è il vero nome della scrittrice), viene scritto nel tra il 1901 e il 1904 e pubblicato nel 1906. Si tratta di un romanzo autobiografico scritto in prima persona e che racconta il viaggio solitario di trasformazione della protagonista e di ricostruzione della sua identità verso la libertà. Sibilla Aleramo "fu la prima donna italiana che iniziò la gran- de rivoluzione femminista" (González, 2009: 292); si potrebbe dire che fu la prima scrittrice italiana del primo Novecento a trattare il tema della donna e per questo motivo viene considerata come una femminista ante litteram. II suo femminismo, né teorico né di azione, è un atteggiamento morale istintivo, un pensiero personale isolato che non si allineò mai con movimenti organizzati ma che fu prima di tutto l'obbedienza a un imperativo interiore che non poteva ignorare. La scrittrice mise in pratica il femminismo in modo autonomo e solitario attraverso una continua e dura lotta personale che la mantenne occupata durante tutta la vita. Si sforzò per cambiare la sua esistenza, oltre a quella di molte altre donne che condividevano le sue stesse inquietudini e lo stesso pensiero, e che avrebbero potuto ricevere da lei un esempio di coraggio e di volontà.

Una donna è un testo fondamentale per conoscere la sua drammatica e singolare vita che gira intorno al lacerante abbandono del figlio Walter che la scrittrice ebbe a diciannove anni (1895). II romanzo documenta il lento e lucido risveglio della coscienza che, ormai matura e forte, ruggisce obbligando la protagonista a quello che sarà l'inizio di un viaggio esistenziale profondo in nome di una battaglia personale verso la creazione di una nuova identità di donna. Il libro, nonostante fosse stato fortemente criticato da alcuni personaggi appartenenti al circolo privato di Sibilla Aleramo, in realtà ottenne fin dall'inizio quella notorietà che forse nemmeno la scrittrice si immaginava; infatti venne accolto con grande clamore dal pubblico, soprattutto femminile, e dalla critica. Una donna viene subito interpretato come il manifesto del femminismo italiano, un femminismo moderno che riflette sulle forme sociali dell'oppressione e della subal- 
ternità. "II nodo è già la questione muliebre, il rovello è la disuguaglianza costruita a partire dal sesso, il nemico è l'immenso sistema che la civiltà ha edificato con l'uso della menzogna" (Folli, 2007: VIII). Nel romanzo viene ribaltato in modo imperioso, come mai prima di allora nessuna scrittrice italiana aveva fatto, il modello di maternità esistente fino a quel momento.

In una serie di articoli pubblicati da "L'Alleanza" nel 1906, Teresa Labriola scriveva: "Dico senza pretese rettoriche, ma con profonda convinzione che la nota fondamentale per la quale il movimento femminile attuale si distingue da quello del passato, è nel sentimento sincero e profondo della maternità" (Buttafuoco, 1988: 12).

Siamo di fronte ad un testo che ha il grande merito di ribellarsi con tutta la voce che la scrittrice ha in corpo a un'idea di maternità intristita da tutti i falsi valori che l'hanno sorretta fino a quel momento: il sacrificio, l'immolazione, il martirologio dell'essere madre. Sibilla Aleramo smantella tenacemente l'antico modello di maternità imposto dalla cultura da cui proviene e in cui vive e che è stato seguito anche dalla propria madre ${ }^{3}$, e ne propone uno nuovo che l'accompagnerà fino alla fine dei suoi giorni. Sibilla pretende di es-

3. Ernesta Cottimo, madre della scrittrice, viene descritta nel romanzo Una donna come una madre passiva e debole, abnegata dal ruolo di madre e di sposa tradita più volte dal marito. Essa, sola e remissiva, fu sempre a contatto con una forma di nevrastenia e di depressione che la porteranno più volte a dei tentativi di suicidio; finirà per ammalarsi e morirà dopo aver passato molti anni in manicomio. Sibilla fin da bambina soffrì molto per questa madre, nei confronti della quale nutrirà per sempre dei sentimenti di disappunto $\mathrm{e}$ di quasi ostilità, in quanto incapace di essere una donna autonoma e realizzata sul piano sia personale che sociale. sere una madre-donna diversa, reinventata, ricostruita, ripensata; intendendo per madre la sostanza più intima dell'essere donna; il suo bisogno più naturale, la sua condizione più primitiva. II concetto di madre e di donna in Sibilla si fondono: la madre mai potrebbe annullare la donna e nemmeno la donna sarebbe capace di eliminare la sua essenza di madre. Sibilla per poter abbattere il modello di madre che le è stato insegnato e poterne vivere un altro, deve partire da zero; da se stessa, dal suo essere donna, rifiutando tutta una serie di cliché e di retaggi culturali che le impedirebbero di rinnovarsi su entrambi i piani. "La donna maturata deve morire perché un'altra possa rinascere" (Folli, 2007: XVII).

In quest'ottica la scrittrice riflette sul fatto che "se non si costruisce una nuova donna con una nuova identità e dei nuovi modelli o miti a cui guardare non può esistere la nuova madre, quella che prodigiosamente potrà insegnare ai propri figli qualcosa di buono" (Aleramo, 2007: 85). Aleramo più che procedere per teorie e postulati, capisce intuitivamente l'errore di vivere, sia come donna che come madre, una vita votata a una serie infinita di sogni infranti, di umiliazioni, di sacrifici non riconosciuti, perché fin da bambina assiste al misero spettacolo che le viene offerto quotidianamente, quello di una madre assente e passiva che ha lasciato morire la donna che è in lei.

Nel romanzo Sibilla racconta la sua storia di donna attraverso la narrazione di numerosi fatti privati ed esperienze personali drammatiche che porteranno la protagonista-donna-scrittrice a scegliere un cammino di emancipazione e di libertà dopo aver seguito l'imperativo ibseniano della fedeltà alla propria legge: la fedeltà alla 
vita ${ }^{4}$. Sibilla sente la necessità di mostrare per la prima volta l'anima femminile moderna e "solo una donna con quella storia potrà tramutarne l'essenza in arte, e sarà il libro" (Folli, 2007: XVII).

II modello che Sibilla in parte segue è il romanzo di formazione, il Bildungsroman ottocentesco che senza dubbio conosceva molto bene e che trasforma la protagonista in eroina dopo un susseguirsi di avvenimenti decisivi per il cambiamento esistenziale.

Una donna scorre su due binari: quello del racconto di una vita, la sua, e quello della testimonianza di un itinerario di rigenerazione: la scrittura. (Zancan, 1998: 53).

La prima parte del libro racconta la fanciullezza e l'adolescenza della protagonista che scorrono a Porto Civitanova, nelle Marche, luogo legato al lavoro del padre, dove è ambientata la vicenda del romanzo. I primi due capitoli si appoggiano sull'affermazione dell'incipit: " La mia fanciullezza fu libera e gagliarda" (Aleramo, 2007: 1). Fin da ragazzina il suo modo di essere desta lo stupore degli uomini, ancora abituati a un modello femminile diverso, assai più contenuto e non così libero ed audace; si delinea così un tema fondamentale che sottolinea, fin dall'inizio del romanzo, la differenza tra i sessi espresso dall'atteggiamento maschile nei confronti della donna quando quest'ul-

4. Nora, la protagonista di Casa di bambola di H. Ibsen, dopo un'esistenza vissuta all'ombra del marito che la infantilizza con frivoli soprannnomi e la sminuisce nel suo ruolo di donna-madre-bambina che gioca con i figli come se fossero bambole, arriva a maturare una coscienza di se stessa e della sua posizione nel mondo come donna autonoma di pensiero e libera, e alla fine del suo percorso, arriverà a non accettare più nessuna legge su cui non abbia prima riflettuto autonomamente. tima ritorna verso casa, dopo aver lavorato nella fabbrica e passa davanti a un gruppo di uomini seduti a un caffè: "[...] Sentivo che da una parte destavo la loro curiosità, dall'altra offendevo la loro abitudine di veder le fanciulle passar timide, guardinghe e lusingate dai loro sguardi" (Aleramo, 2007: 15). Questo suggerire fin dall'inizio una forte consapevolezza del suo essere donna e un atteggiamento non di sottomissione ma di fierezza davanti al mondo, anticipa in un qualche modo la posizione in cui si schiererà la protagonista di fronte alla vita: sempre libera, forte, diversa, di fronte a un universo maschile giudicante e severo.

Nei capitoli successivi di Una donna, vengono raccontati diversi fatti privati che segneranno in modo decisivo e permanente l'esistenza di Rina Faccio, non ancora Sibilla Aleramo ${ }^{5}$. II terzo capitolo è significativo perché segna il passaggio dall'infanzia alla vita adulta, con svariate esperienze femminili che portano la protagonista a vivere circostanze volte al dolore, all'apatia, perfino alla follia. Ci viene narrata la violenza sessuale sofferta da adolescente da colui che ne diverrà in futuro il marito, Uldarico Pierangeli, e anche la successiva rimozione del malfatto a cui segue più avanti il dolore legato alla delusione nei confronti dell'adorato padre che ci viene presentato fin da subito come una figura forte e positiva ma che in seguito, inaspettatamente, dimostrerà di essere non diverso da tutti gli altri; egli infatti tradirà la moglie portandola sull'orlo della pazzia e per la ragazzina gli anni a venire

5. Sibilla Aleramo è il nome che le darà Giovanni Cena, compagno sentimentale della scrittrice durante la sua seconda esistenza che si inaugurerà quando nel 1902 abbandonerà il nido coniugale $\mathrm{e}$ il figlio Walter e da Portocivitanova Marche raggiungerà Milano. 
saranno disincantati e tristi. II padre, l'unico modello che guidava i suoi passi, crolla inesorabilmente e le lascerà un vuoto incolmabile che non l'abbandonerà mai più.

II rapporto con la madre, figura umiliata e smarrita, è difficile e irrisolto, frustrato per la sua incapacità di comprenderla ed aiutarla e di dimostrarle qualsiasi manifestazione di solidarietà filiale. II gesto del suicidio materno contribuirà allo stato di costante affliggimento della protagonista: "Sempre, latente, era il terrore di vederla ripresa dalla necessità d'un qualche folle e fatale atto. E ancora, più triste che mai, il dubbio di non amarla quanto avrei dovuto e voluto, di essere impotente di fronte alla sua infelicità!" (Aleramo, 2007: 22). La madre appartiene alla schiera delle madri «vili», le prime vittime del sistema patriarcale (Arriaga Flórez, 2016: 147) e la protagonista non troverà mai in lei un modello femminile da cui imparare a vivere come donna, verso il quale guardare come se di uno specchio si trattasse: "La sua debolezza, la sua rinuncia alla lotta mi esacerbavano tanto più in quanto ero costretta a riconoscermi ora dei punti di contatto con lei nella mia rassegnazione al destino" (Aleramo, 2007: 39).

La follia che colpisce la madre dopo i ripetuti tradimenti del marito porta la protagonista a provare quasi ribrezzo verso questa figura larvale che non è più in grado di vivere in modo degno:

Dinanzi a quella miseria umana che mi ricercava nel mezzo della notte, ebbi una rivolta selvaggia di tutto l'essere... Tremavo, in preda anch'io alla febbre... E lanciai alla sventurata parole acerbe, folli quasi come le sue... Oh, mia madre! ... E per l'amore di un uomo che non la meritava più! [...] Amare e sacrificarsi e soccombere! Questo era il destino suo e forse di tutte le donne? (Aleramo, 2007: 40- 41).
In parallelo al dramma della madre ne scorre un altro che diventa il nodo centrale intorno a cui si svolge il racconto: quello dello stupro avvenuto in fabbrica che ribadisce ancora una volta il tema della violenza maschile nei confronti delle donne nel contesto di "una società che le eleva come madri e muse ma che allo stesso tempo le emargina, le maltratta e talvolta le vende e le compra" (Martínez Garrido, 2009: 132).

La volgare e prepotente figura di quello che sarà il marito della protagonista, obbligherà questa povera ragazzina a una serie di pratiche amorose che, se all'inizio sembravano essere sincere e ispirate da un puro sentimento amoroso, presto si riveleranno meschine, violente, sfrenate:

Così, sorridendo puerilmente, accanto allo stipite di una porta che divideva lo studio del babbo dall'ufficio comune, un mattino fui sorpresa da un abbraccio insolito, brutale: due mani tremanti frugavano le mie vesti, arrovesciavano il mio corpo fin quasi a coricarlo attraverso uno sgabello mentre istintivamente si divincolava. Soffocavo e diedi un gemito ch'era per finire in urlo, quando l'uomo, premendomi la bocca, mi respinse lontano. Udii un passo fuggire e sbattersi l'uscio. Barcollando mi rifugiai nel piccolo laboratorio in fondo allo studio. Tentavo ricompormi, mentre mi sentivo mancare le forze; ma un sospetto oscuro mi si affacciò. Slanciatami fuor della stanza, vidi colui, che m'interrogava in silenzio, smarrito, ansante. Dovevo esprimere un immenso orrore, poiché una paura folle gli apparì sul volto, mentre avanzava verso di me le mani congiunte in atto supplichevole... [...] Appartenevo ad un uomo, dunque? Lo credetti dopo non so quanti giorni d'uno smarrimento senza nome (Aleramo, 2007: 26-27).

Sibilla, in seguito a questo dramma, mette in atto un processo di rimozione che la por- 
ta a dimenticare il fatto avvenuto o quanto meno a mal interpretarlo; non le esce di bocca nemmeno una parola, neppure con il colpevole, e fin da subito si sente spogliata, oltre che della sua verginità, della sua personalità, di ciò che era stata fino a un anno innanzi. La piena coscienza dello stupro, Rina-Sibilla la raggiungerà solo una volta che lo racconterà nel romanzo (Folli, 2007: XV). Come lei stessa scrive: "La mia personalità fin allora così libera, dinanzi alla memoria del fatto ch'io consideravo irreparabile, insorgeva a tratti, ma soltanto per farmi più sentire la sconfitta patita" (Aleramo, 2007: 31).

L'amante retrogrado presto dimostrerà di essere un maltrattatore che porta la protagonista a intraprendere una vita che non ha scelto ma nella quale è precipitata senza quasi essersene accorta: quella del matrimonio forzato, dopo lo stupro. Nascerà poi un figlio e per lei inizieranno anni di buia disperazione, di perdita d'identità, di annullamento e di abnegazione; solo la maternità, in un primo momento, vissuta spontaneamente come sentimento di vero amore, umano e viscerale, e più avanti la scrittura, riusciranno a darle un po' di sollievo in mezzo a tanto dolore.

II marito è soddisfatto della trasformazione della sua donna il cui carattere diventa ogni giorno più remissivo, e il suo maschilismo tirannico riesce a relegarla in una solitudine che fa di lei un essere piccolo e privo di forza. Dopo la nascita del figlio, il marito continua ad esercitare su di lei violenze fisiche e psicologiche e la prima parte del libro, fino al nono capitolo, si chiude con l'intenzione di suicidio della protagonista, ormai esausta di fronte a tanta sofferenza: "Quell'uomo mi aveva soggiogata per tante settimane, aveva saputo imporsi al mio pensiero... Perché? Perché ero sola, disarmata, asse- tata ed anelante..."(Aleramo, 2007: 61). Lui, miserabile, brutale, egoista, autoritario, al primo accenno d'indipendenza della moglie, riesce a far sì che lei torni indietro, che rinunci a se stessa; la donna ormai è accecata dalla disperazione, la pazzia sembra invaderla. La protagonista è già intenta all'ascolto della sua rivolta interiore, il cambiamento è già iniziato e l'intenzione di portarlo avanti, attraverso un'azione radicale, ormai è sancita. Se la maternità in un primo momento avrebbe potuto legarla ancora a questo mondo, ora non più; nemmeno il figlio riesce a farla sentire un individuo degno di vivere una vita vera e non ha il potere sufficiente per risvegliare la sua antica personalità: forte, libera, selvaggia, ormai dimenticata:

Ero mai stata donna, fino allora? No, neppure partorendo, neppure nutrendo con il mio latte mio figlio ero pervenuta a sentire in me la ragione della mia esistenza e quella del mondo. II mio bambino l'avevo adorato, ma come una parte di me, più arcana, che m'attaccava, sì, viepiù alla terra, ma ancora interrogando, senza il mio consenso, senza l'accordo della mia volontà con la volontà della vita: mio figlio non era frutto d'amore, non era neanche, povero piccolo palpitante cuore del mio cuore, non era figlio di tutta me, era nato da me prima che fossi io stessa tutta nata, prima che io fossi veramente fiorita. Come una grande rosa al sole la donna s'apriva ora, e il profumo n'andava lontano (Aleramo, 1985: 26).

II ricordo della sua infanzia, della sua "fanciullezza libera e gagliarda", la tormenta e le suscita l'eterno desiderio di ritornare a quello stato d'animo che i drammi della sua vita hanno allontanato per sempre; rivuole i più begli anni della giovinezza che la precoce e dura realtà hanno mortificato facendola divenire madre prima del tempo, quando ancora necessariamente 
avrebbe dovuto formarsi come donna, fiorire e maturare (Zancan, 2000: 126). La consapevolezza che lo stupro abbia impedito l'esprimersi della sua naturale gioia e vigore, del suo incanto, la abbatte come la più profonda delle ingiustizie e il dolore diventa un urlo che non riesce ancora a liberarsi in tutta la sua potenza ma che è già pensiero, corpo, sangue.

Raccontare la vita significa rendere fecondo il dolore: Sibilla chiede alla scrittura di cambiare la sua esistenza e la rinascita avviene con e attraverso il libro; la nuova donna inizia quando la sua storia viene scritta. Sibilla deciderà di mettere una pietra sul sepolcro della sua prima esistenza, altrimenti ormai votata per sempre alla rinuncia a tutte le cose buone. Nel passaggio dalla sua vita misera a quella nuova così tanto anelata, disgraziatamente nemmeno il figlio trova un posto, non può essere salvato nemmeno lui, poiché fare ciò equivarrebbe a rinunciare a tutto, perfino alla verità e alla libertà della sua anima. Solo salvando se stessa potrà poi, un giorno, salvare lui.

II discorso sulla maternità che porta avanti Aleramo è di grande importanza in quanto viene preso ed accettato come manifesto del femminismo che si oppone al concetto di maternità sacrificale (Martínez Garrido, 2019: 63). Aleramo sostiene una poetica della maternità nel quale, come dicevamo precedentemente, la donna-madre deve esistere allo stesso tempo per se stessa e per la sua creatura costruendosi come 'persona umana', buona, forte e libera, con una propria identità femminile che la allontani per sempre dal ruolo di vittima sacrificale, martire della maternità. Pensiamo alle «madri mediocri» che sono state allontanate dalla loro natura femminile sacra che Pasolini identifica con il sentimento, la passione («reali richiami del cuore») l'amore, la cura («che non hanno avuto per voi mai una parola d'amore») e che riproducono l'identità patriarcale che, con le sue norme, stabilisce «un unico, nudo significato», un'identità obbligatoria, che non può essere pensata altrimenti. La sopraffazione del maschile sul femminile è raffigurata nelle «madri mediocri», che imparano "con umiltà da bambine» dai propri figli (Arriaga Flórez, 2016: 147). A questo riguardo riportiamo un passaggio di Una donna:

E incominciai a pensare se alla donna non vada attribuita una parte non lieve del male sociale. Come può un uomo che abbia avuto una buona madre divenir crudele verso i deboli, sleale verso una donna a cui dà il suo amore, tiranno verso i figli? Ma la buona madre non deve essere, come la mia, una semplice criatura di sacrificio: deve essere una donna, una persona umana. E come può diventare una donna, se i parenti la danno, debole, incompleta a un uomo che non la riceve come sua eguale; ne usa come di un oggetto di proprietà; le dà i figli con i quali l'abbandona sola, mentr'egli compie i suoi doveri sociali, affinché continui a baloccarsi come nell'infanzia? Perché nella maternità adoriamo il sacrificio? Donde è scesa a noi questa inumana idea dell'immolazione materna? Di madre in figlia, da secoli, si tramanda il servaggio. Ė una mostruosa catena. Tutte abbiamo a un certo punto della vita, la coscienza di quel che fece per il nostro bene chi ci generò; e con la coscienza di rimorso di non aver compensato adeguatamente I'olocausto della persona diletta. Allora riversiamo sui nostri figli quanto non demmo alle madri, rinnegando noi stesse e offrendo un nuovo esempio di mortificazione, di annientamento. Se una buona volta la catena fatal si spezzasse, e una madre non sopprimesse in sé la donna, e un figlio apprendesse della vita di lei un esempio di dignità? (Aleramo, 2007: 85). 


\section{Il viaggio esistenziale di ricostruzione verso una nuova identità di donna: il sacrificio umano della maternità}

La seconda parte del libro si snoda attorno alla trasformazione della protagonista e definisce i tratti di quella nuova figura di donna la cui nascita è ormai legata alla scrittura del libro: "Vivere! Ormai lo volevo, non più solo per mio figlio, ma per me, per tutti" (Aleramo, 2007: 86).

Inizia per la protagonista un nuovo itinerario di crescita e di formazione, scorrono mesi durante i quali vive di meditazioni, letture e dell'amore di suo figlio; nasce una nuova donna attenta alla sua legge e ai suoi bisogni. La protagonista sente di dover recuperare la parte più intima e profonda del suo essere per potersi sentire in pace con se stessa e la scrittura è il mezzo per arrivare alla rinascita come 'individuo umano'. II figlio l'accompagna in questo processo di liberazione e il sogno grandioso di una fusione assoluta, il miracolo di un'unità a due che aveva avvolto la sua infanzia, si ripete nell'amore per il figlio; il rapporto con il bimbo si sovrappone all'antico rapporto con il padre e ne rinsalda idealmente la violenta frattura, risarcendo l'abbandono e la sconfitta patiti. II figlio e la 'bambina' sembrano confondersi e, per un breve tempo, questo equilibrio sembra funzionare. L'illusione di una possibile continuità della madre nel figlio si svela però come progressiva morte fine a se stessa già alla fine del settimo capitolo (Zancan, 1998: 203):

In verità, al di fuori della somma di energie ch'io spendevo attorno al bambino, era in me un'incapacità sempre maggiore di vedere, di volere, di vivere: come una stanchezza morale si sovrapponeva a quella fisica, lo scontento di me stessa, il rimprovero della parte migliore di me che avevo trascurata, di quel mio io profondo e sincero, così a lungo represso, mascherato. Non era un'infermità, era la deficenza fondamentale della mia vita che si faceva sentire. In me la madre non s'integrava nella donna: e le gioie e le pene purissime in essenza che mi venivano da quella cosa palpitante e rosea, contrastavano con un'instabilità, un'alternazione di languori e di esaltamenti, di desideri e di sconforti, di cui non conoscevo l'origine e che mi facevano giudicare da me stessa un essere squilibrato e incompleto (Aleramo, 2007: 51 ).

La maternità diventa un atto creativo teso a non nascondere la donna dietro alla madre e diventa l'impulso generatore di una vita fatta di spinte e di accelerate verso una coscienza di se stesse come madri ma prima ancora come donne ancora capaci di affermarsi oltre la maternità e ad assicurarsi quell'appagamento che oltre ad arricchire la madre, sarà anche a beneficio della propria creatura: "Era quel suo sorriso il premio, l'approvazione quotidiana del mio sforzo. Pareva dicesse: lo sento che tu lavori anche per me, mamma, sento che tu fiorisci, ti espandi, vivi, e perciò diventi forte e buona, e mi prepari un'esistenza forte e buona..." (Aleramo, 2007: 89).

Ciò nonostante Sibilla non riesce a svolgere il suo ruolo di madre e il conflitto che nasce da questa consapevolezza è espresso con grande forza di sentimento:

Mancava a me la volontà continua della vera educatrice, la serenità di spirito per guidare la piccola esistenza; non potevo assorbirmi intera nella considerazione dei suoi bisogni, prevenirli, soddisfarli. In certi istanti per questa consapevolezza mi odiavo. Che miserabile ero dunque se non riuscivo, una 
volta accettato il sacrificio della mia individualità, a dimenticare me stessa, a riportare integre le mie energie su quella individualità che mi si formava a lato? (Aleramo, 2007: 143).

Nella terza ed ultima parte del libro la protagonista, giunta alla fine del percorso della sua rinascita personale con tutte le battaglie che questo ha comportato, arriva alla decisione sofferta della necessaria fuga dal nido coniugale e alla dolorosissima separazione dall'amato figlio.

Le tematiche della maternità e della scrittura segnano il doppio itinerario di Sibilla persona e Sibilla scrittrice, in cui la scrittura prende il sopravvento sul figlio e si presenta come l'esperienza che sancisce il passaggio verso la sua seconda esistenza. II problema posto dal romanzo nasce dalla riflessione che se una donna è pronta a svolgere compiutamente la propria individualità, di cui la maternità è parte integrante, allora questa donna recide da sé qualcosa che è pregiudiziale proprio a quello svolgimento, ovvero, il figlio (Folli, 2007: XIII). La scelta estrema che porta la protagonista a non sopprimere in se la donna, forse insegnerà al figlio qualcosa di importante e gli offrirà un esempio di dignità per la vita.

L'immagine della nuova donna che ha riconosciuto la propria legge porta a termine un itinerario prestabilito; la madre che abbandona il figlio si prepara al destino di essere "un'idea vivente", di incarnare una "verità" da esprimere, per la salvezza degli altri, in "una parola memorabile" (Zancan, 1998: 209):

A tratti, un senso di ammirazione quasi da estranea mi prendeva per il cammino da me percorso; avevo la rapida intuizione di significare qualcosa di raro nella storia del sentimento umano, d'essere tra i depositari d'una verità manifestantesi qua e là a dolorosi privilegiati... E, pensosa, mi chiedevo se sarei riuscita un giorno ad esprimere per la salvezza altrui una parola memorabile (Aleramo, 2007: 149).

L'ultimo momento d'intimità tra il bimbo nel suo letto e la mamma al suo fiancoe è atroce:

Accesi la lampada, la coprii. Un fruscìo. "Mamma?" Mi slanciai sul lettuccio: posò la mano nella mia e si riaddormì. Rimasi senza muovermi, quasi senza respiro. Mezzanotte. Mancavano tre ore. Le ginocchia mi si piegarono. Seduta sulla poltrona sentivo il freddo invadermi, e raccoglievo tutto il mio calore, gli occhi chiusi, ritirando la mia mano per non agghiacciare la manina. $E$ d'un tratto sentii tutte le mie forze fondersi: mi assopivo? Ero tanto stanca: non avrei potuto partire...Scoccarono le tre. Balzai in piedi. Mi misi il mantello e m'appressai all'uscio. Poi tornai al letticciuolo, svegliai il bimbo: "Vado", gli dissi piano, "è già l'ora; sii buono, sii buono, voglimi bene, io sarò sempre la tua mamma..."e lo baciai senza poter versare una lagrima, vacillando; e ascoltai la vocina sonnolenta che diceva: "Sì, sempre bene... Manda il nonno a prendermi, mamma... Star con te...". Si voltò verso il muro tranquillo. Allora, allora sentii che non sarei tornata, sentii che una forza fuori di me mi reggeva, e che andavo incontro al destino nuovo, e che tutto il dolore che mi attendeva non avrebbe superato quel dolore (Aleramo, 2007: 160-161).

La donna prende un treno nel cuore della notte; abbandona il figlio con la speranza che un giorno la legge potrà aiutarla a recuperarlo. II tempo passa e si rende conto che nessuno può restituirle il suo bimbo: la legge non la protegge e perfino suo padre, ormai esausto, rinuncia alla lotta, abbandonando ogni intervento per recuperare il nipote. L'unica consolazione 
di questa madre rimane la speranza che il figlio non la dimentichi e che forse un giorno provi il desiderio di partire per andarla a cercare; durante questa insopportabile e inumana attesa, l'unica cosa che spera è che le sue parole lo raggiungano e che il suo libro sia servito per lui, per raccontargli il suo cuore, la sua pena, la verità, il suo amore; l'amore di una madre che ha dovuto abbandonarlo non per disamore ma per poter salvare entrambi da una prigione di menzogne e di dolore:

Partire, partire per sempre. Non ricadere mai più nella menzogna. Per mio figlio più ancora che per me! Soffrire tutto, la sua Iontananza, il suo oblìo, morire, ma non provar mai il disgusto di me stessa, non mentire al fanciullo, crescendolo, io, nel rispetto del mio disonore! (Aleramo, 2007: 157).

II progetto egotico dell'Aleramo rivela al suo meglio la propria anarchia, esalta un'incapacità d'insubordinazione alla legge in favore di un personale, esclusivo codice che ammette come a "rinnovare il respiro del mondo non valgano i Cesari portatori di guerra, ma solo la gloriosa solitudine di qualche genio, I'oltranza di qualche libero esploratore" (Guerricchio, 1997: IX).

La femminista Sibilla Aleramo con Una donna, ci ripropone ancora oggi un modello di comportamento al femminile, un decisivo atto radicale di libertà, coraggioso e tenace, attraverso un capolavoro che continua ad offrire, soprattutto alle donne contemporanee, un esempio di eroicità senza pari.

\section{Riferimenti bibliografici}

Addis Saba, M., La Corporazione delle donne. Ricerche e studi sui modelli femminili nel ventennio fascista, Vallecchi, Firenze, 1988.
Aleramo, S., Amo dunque sono, FeltrineIli, Milano, 1998.

Aleramo, S., Andando e stando, Feltrinelli, Milano, 1997.

Aleramo, S., Una donna, Feltrinelli, Milano, 2007.

Aleramo, S., Un viaggio chiamato amore. Lettere 1916-1918, Valter Casini Editore, Milano, 2004.

Aleramo, S., I/ passaggio, Serra e Riva Editori, Milano, 1985.

Aleramo, S., Orsa minore, Feltrinelli, Milano, 2002.

Aleramo, S., Selva d'amore, Mondadori, Milano, 1947.

Aleramo, S., I/ frustino, Mondadori, Milano, 1932.

Aleramo, S., Endimione, Stock, Roma, 1923.

Aleramo, S., Dal mio diario 1940-44, Tumminelli, Roma, 1945.

Arriaga Flórez, M., "La perspectiva pragmática del texto autobiográfico: Cuando el diario se convierte en autobiografía: Sibilla Aleramo", Philologia hispalensis, n 6 , 1991, pp. 127-134.

Arriaga Flórez, M., "La Continuità Dello Specchio: Sibilla Aleramo", en P. Zaccaria, P. Calefato (coord.) Segni Eretici. Scrittura di Donne tra Autobiografía, Etica e Mito, Adriatica, Bari, 1993, pp. 71-82.

Arriaga Flórez, M., Mi amor, mi juez. A/teridad autobiográfica femenina, Anthropos, Barcelona, 2001.

Arriaga Flórez, M., "Pier Paolo Pasolini: le madri vili generano la società borghese", Cuadernos de Filología italiana, $\mathrm{n}^{\circ} 23$, 2016, pp. 141-153. 
Austen, J, Orgoglio e pregiudizio, Mondadori, Milano, 1979.

Bourdieur, P., I/ dominio maschile, Feltrinelli, Milano, 1998.

Braidotti, R., madri, mostri e macchine, manifesto libri srl., Roma, 1996.

Braidotti, R., Sujetos nómades. Corporización y diferencia sexual en la teoría feminista contemporánea, Paidós, Buenos Aires, 2000.

Brontë, C., Jane Eyre, Einaudi, Torino, 2010.

Buttafuoco, A., Zancan, M., Svelamento. Sibilla Aleramo: una biografia intellettuale, Feltrinelli, Milano, 1988.

Buzzatti G., Salvo A., El cuerpo-palabra de las mujeres. Los vínculos ocultos entre el cuerpo y los afectos, Cátedra, Madrid, 2001.

Ciplijauskaité, B., La novela femenina contemporánea. (1970-1985). Hacia una tipología de la narración en primera persona, Anthropos, Barcelona, 1994.

Conti, B., Morino, A., Sibilla Aleramo e il suo tempo. Vita raccontata e illustrata, Feltrinelli, Milano, 1981.

Contorbia F., Melandri L., Morino A., SibiIla Aleramo. Coscienza e scrittura, Feltrinelli, Milano, 1986.

Debenedetti, G., /l romanzo del Novecento, Garzanti, Milano, 2006.

De Ceccatty, R., Sibilla Aleramo, Éditions Du Rocher, Monaco, 1992.

De Céspedes, A., Quaderno proibito, ॥ Saggiatore, Milano, 2006.

De Giorgio, M., Le italiane dall'Unità ad oggi. Modelli culturali e comportamenti sociali, Laterza, Roma-Bari, 1992.
De Luna, G., Donne in oggetto, Bollati Boringhieri, Torino, 1995.

Duby, G., Perrot, M., Storia delle donne, II novecento, Laterza, Roma-Bari, 2007.

Folli, A., Penne leggère, Guerini e Associati, Milano, 2000.

Guidi, L., Scrittura femminile e Storia, ClioPress, Napoli, 2004.

González, I., "La revolucionaria e insumisa Sibilla Aleramo: II passaggio", en E. González de Sande., A. Cruzado Rodríguez (coord.) Las Revolucionarias. Literatura e insumisión femenina, Arcibel Editores, Sevilla, 2009, pp. 291-302.

Héritier F., Maschile e femminile, Editori Laterza, Roma-Bari, 2000.

Ibsen, H., Casa de muñecas, Ediciones Cátedra, Madrid, 2007.

Lispector, C., La scoperta del mondo. 1967-1973, La Tartaruga, Milano, 2001.

Maddamma, M., Anime estreme, Vallecchi, Firenze, 2009.

Madeo, L., Donne cattive, La Tartaruga, Milano, 1999.

Martínez Garrido, E., "Bildungsroman y crítica de género. Novela rosa y narrativa de mujeres", Cuadernos de Filología Italiana, $n^{\circ}$ VII, 2009, pp. 529-546.

Martínez Garrido, E., "La violencia contra las mujeres al renacer de la nueva identidad", en E. Martínez Garrido (coord.) Transmisión y apología del uso de la violencia contra las mujeres: refranes, dichos y textos literario, Publicaciones de la UCM, Madrid, 2009, pp. 128-148.

Martínez Garrido, E., "Una donna di Sibilla Aleramo: un viaggio verso l'identità", en G. Ioli (coord.) Sibilla Alleramo una donna del Novecento, Interlinea, Novara, 2019, pp. 61-69. 
Melandri, L., "Sibilla Aleramo. Una coscienza femminile anticipatrice", en G. Ioli (coord.) Sibilla Aleramo una donna del Novecento, Interlinea, Novara, 2019, pp. 33-43.

Messina, M., Piccoli gorghi, Sellerio editore, Palermo, 1988.

Messina, M., La casa nel vicolo, Sellerio editore, Palermo, 1999.

Morante, E., La storia, Einaudi, Torino, 1995.

Morante, E., Diario 1938, Einaudi, Torino, 2005.

Neera, Duello d'anime, Treves, Milano, 1911.

Omero, Odissea, Einaudi, Torino, 1963.

Paz, O., La llama doble. Amor y erotismo, Editorial Seix Barral, Barcelona, 1997.

Rampello, L., I/ canto del mondo reale, II Saggiatore, Milano, 2005.

Romano, L., Maria, Einaudi, Torino, 1995.

Romano, L., Le parole tra noi leggere, Einaudi, Torino, 1996.

Rosa, G., Cattedrali di carta, II Saggiatore, Milano, 2006.

Sackville-West, V., Adorata creatura, La Tartaruga, Milano, 2000.

Sánchez-Pardo González, E., “¡Hacia un feminismo posmoderno? Reflexiones en torno al género y la lectura en la posmodernidad", Estudios ingleses de la UCM, $\mathrm{n}^{\circ} 1$, 1993, pp. 143-158.

Vivanti, A., Vae Victis, Quintieri, Milano, 1917.

Woolf, V., Orlando, Mondadori, Milano, 1993.

Woolf, V., Una stanza tutta per sé, Feltrinelli, Milano, 2011.
Woolf, V., Un collegio di ragazze, Garzanti, Milano, 2019.

Yourcenar, M., Pellegrina e straniera, Einaudi, Torino, 1990.

Zambrano, M., Persona y Democracia. La historia sacrificial, Anthropos, Barcelona, 1988.

Zambrano, M., All'ombra del Dio sconosciuto, Pratiche Editrice, Milano, 1997.

Zancan, Marina, "Una donna di Sibilla Aleramo", en A. Asor Rosa (coord) Letteratura italiana, Le opere, IV/I II Novecento. L'età della crisi, Einaudi, Torino, 1995, pp. 101-143.

Zancan, M., /l doppio itinerario della scrittura, Einaudi, Torino, 1998. 